\title{
Balkanologie
}

Balkanologie Revue d'études pluridisciplinaires

Vol. II, nº 2 | 1998

Volume II Numéro 2

\section{L'Église orthodoxe grecque confrontée à la modernité : crispations et résistances}

Jacky Pruneddu

\section{(2) OpenEdition}

\section{Journals}

Édition électronique

URL : http://journals.openedition.org/balkanologie/257

DOI : 10.4000/balkanologie. 257

ISSN : 1965-0582

Éditeur

Association française d'études sur les Balkans (Afebalk)

Édition imprimée

Date de publication : 1 décembre 1998

ISSN : 1279-7952

\section{Référence électronique}

Jacky Pruneddu, "L'Église orthodoxe grecque confrontée à la modernité : crispations et résistances », Balkanologie [En ligne], Vol. II, n² 2 | 1998, mis en ligne le 02 juin 2008, consulté le 17 décembre 2020. URL : http://journals.openedition.org/balkanologie/257 ; DOI : https://doi.org/10.4000/balkanologie 257

Ce document a été généré automatiquement le 17 décembre 2020.

(c) Tous droits réservés 


\title{
L'Église orthodoxe grecque confrontée à la modernité : crispations et résistances
}

\author{
Jacky Pruneddu
}

1 Les réflexions qui seront faites ici s'appuieront d'abord sur les divers textes, revues et publications, officielles ou semi-officielles, de l'Église orthodoxe grecque concernant les problèmes qu'elle rencontre dans ses inévitables contacts avec la modernité. D'autre part, considérant les menaces de déchristianisation, parfaitement perçues par l'Église nationale, j'ai tenté de savoir si cette déchristianisaion pouvait être considérée comme en marche chez les jeunes étudiants d'aujourd'hui. Le questionnaire avec lequel j'illustrerai certaines considérations et qui portait sur environ 600 jeunes des universités d'Athènes et de Volos ${ }^{1}$ m'a persuadé de ce dont mon long séjour dans mon pays d'adoption m'avait déjà à demi convaincu : l'attachement à l'orthodoxie (qui peut fort bien s'accompagner d'absence de foi) est profondément lié, et c'est même là, peutêtre, la forme la plus répandue, à l'attachement à l'identité nationale. Ceci a été bien des fois souligné par les chercheurs ${ }^{2}$ et en a amené plus d'un à considérer que "l'orthodoxie est actuellement une condition sine qua non pour la Grèce moderne »" En ce sens, l'Église grecque est, pourrait-on dire, forte justement de ce qui est également sa faiblesse: son caractère essentiellement national, et qui remonte très loin dans l'histoire, dont elle n'a pas pu se détacher ${ }^{4}$ et qu'elle s'efforcera sans doute de conserver malgré la situation d'Église transnationale qu'elle retrouve aujourd'hui avec la nouvelle situation dans les pays de l'Europe de l'Est et des Balkans.

Un autre danger menace l'Église orthodoxe grecque, du moins à ses yeux: l'appartenance du pays à l'Europe communautaire et les «dangereux risques d'aliénation et de syncrétisme religieux $»^{5}$ que cela représente. Bien sûr, la "menace de l'Ouest" n'est pas nouvelle et remonte très loin dans l'histoire. On serait en droit d'évoquer le "patriotisme" byzantin, qui s'est développé, justement, en partie contre les revendications et les agissements de l'Occident, et notamment à la suite des attaques normandes qui «traumatisèrent profondément les chrétiens d'Orient » et du 
« comportement inqualifiable des Latins » lors de la prise de Constantinople en 1204, tout cela provoquant "un sentiment d'humiliation qui se mua en haine ${ }^{6}$ et une véritable "obsession du méchant Latin » portée à son paroxisme dès la fin du XII ème siècle $»^{7}$. Et, même si les anathèmes respectifs qui firent suite au schisme de 1054 ont été réciproquement levés dans les années 1960, la méfiance à l'égard de la soeur ennemie du catholique demeure très vivace.

3 Mais ce n'est pas le catholicisme seul qui représente la menace. En fait, l'Europe (l'Occident) est tombé, aux yeux des milieux orthodoxes, de Charybde en Scylla puisque le mouvement de déchristianisation et la montée de l'athéisme introduisent dans les législations mêmes de ces pays des mesures inacceptables (ou difficilement tolérables) pour la tradition théocratique grecque et s'accompagnent d'une autonomisation désormais quasi-totale du politique et du social. L'Église orthodoxe est donc dorénavant sur la défensive et ne peut que négocier avec les représentants élus du pouvoir politique. Certes, jusque naguère encore, tous les compromis adoptés avaient été relativement favorables à l'Église et pouvaient donc, à ses yeux, être acceptables. Il n'en est plus de même aujourd'hui où les forces "anticléricales" se sont très sensiblement renforcées.

4 Étant expressément désignée comme "religion d'État", l'Église orthodoxe jouit encore de considérables privilèges, et voit même son prosélytisme, non seulement autorisé8, mais encore financé et pris en charge par l'État par le biais de l'obligation de l'enseignement religieux dans les écoles et collèges. Il n'est donc sans doute pas aussi abusif qu'on pourrait le penser de considérer que «nous ne sommes pas un État laïc. Nous sommes une société théocratique modernisée $»^{9}$. De toute évidence en opposition avec les Droits de l'homme, tels, du moins, que les conçoivent aujourd'hui nos sociétés occidentales modernes, il serait étonnant que cette question de l'obligation religieuse à l'école ne fasse pas, un jour ou l'autre, l'objet d'un débat au sein des instances européennes. Et d'autres questions seront sans doute soulevées, qui pourraient mener à une certaine "déchristianisation" de l'État, voire de la société. Des pratiques culturelles comme l'attribution du nom à l'enfant ${ }^{10}$ ou le mariage demeurent, c'est vrai, essentiellement des cérémonies religieuses, tant les pressions sociales sont encore lourdes sur les jeunes, et tant la charge émotionnelle des fêtes religieuses est forte. Cependant, la porte est entrouverte, notamment depuis une loi de 1983 instaurant la possibilité du mariage civil.

5 Autre exemple: les partenaires de la Grèce ne réclameront-ils pas, tôt ou tard, l'abolition de la mention de la religion sur les cartes d'identité, mention que le gouvernement socialiste de 1981-1989 avait proposé de supprimer (il dut faire marche arrière devant la mobilisation du lobby orthodoxe, très présent au Parlement) ? La même mésaventure est arrivée au gouvernement suivant, pourtant conservateur, à la suite de la vive réaction de l'Église ${ }^{11}$. Il n'est pas étonnant que la question soit remise régulièrement sur le tapis, car cette mention de la religion des citoyens est en contradiction évidente avec les divers textes européens sur la défense des libertés individuelles ${ }^{12}$ et empêche les Grecs de voyager en Europe avec une simple carte d'identité. Il est clair, du reste, que les mentalités sont en train d'évoluer et que l'Église se trouve de plus en plus sur la défensive sur cette question, comme l'a montré un débat organisé sur ce point par la chaîne privée de télévision à forte audience, Antenna (« l'heure de vérité » du 2 avril 1997), où le représentant de l'orthodoxie fut conduit à 
déclarer qu'en cas de suppression de la mention de l'appartenance religieuse, l'Église demanderait à ses fidèles de boycotter les nouvelles cartes.

L'Église orthodoxe grecque se trouve ainsi confrontée à ce phénomène (qu'elle a parfaitement perçu) que la sécularisation de la société est amorcée, qu'» un certain ébranlement des fondements est en train de se produire, silencieusement et progressivement $\aleph^{13}$, que les valeurs des nouvelles générations s'éloignent de plus en plus des siennes, que le développement de la conscience éthique s'oppose de plus en plus aux injonctions de la morale chrétienne et que la séparation du spirituel et du temporel ne saurait conduire qu'à l'effacement progressif de son magistère politicoculturo-moral.

$7 \quad$ ll est indéniable que l'on assiste à une érosion des croyances religieuses dans le monde occidental contemporain, et il était quasiment inévitable que l'orthodoxie suive, elle aussi, le mouvement de récession largement amorcé dans la Chrétienté, disons depuis le XVIII ${ }^{\text {ème }}$ siècle, et qui voit s'affaiblir à la fois la pratique religieuse et les valeurs normatives de la morale chrétienne, au profit d'une éthique fondée sur la liberté de penser et d'agir, et l'importance du sacré dans la vie quotidienne. Cependant, dès lors que, même dans les pays à dominance chrétienne de l'Europe latine, le christianisme a su maintenir une forte prééminence culturelle, on peut penser que l'orthodoxie grecque conservera plus longtemps encore cette prépondérance, d'autant plus que nationalisme et orthodoxie sont ici étroitement imbriqués. L'Église grecque, que l'on peut qualifier de garante de la "grécité" pendant les quatre siècles où la Grèce pouvait être considérée comme une nation sans État, en maintenant (du moins est-ce l'image qui en est donnée dans l'histoire scolaire et officielle ${ }^{14}$ ) cet élément déterminant de la culture et de la conscience nationale qu'est la langue, continue de toucher également les dividendes de son investissement supposé du siècle passé dans la lutte de libération nationale, ayant su se présenter rétrospectivement comme le catalyseur des forces patriotiques face à l'occupant étranger.

\section{Remarques sur le questionnaire}

8 Tout questionnaire en matière d'adhésion ou de pratiques religieuses se condamne à une faible crédibilité, tant les censures conscientes ou inconscientes en ce domaine sont particulièrement rigides. C'est, du reste, en matière de religion, irrationnelle par définition, que les réticences sont les plus vives contre toute approche à prétention scientifique, contre tout regard critique, voire simplement neutre. De plus, toute classification entre croyants est toujours délicate, qu'il s'agisse de la fréquentation religieuse $\mathrm{e}^{15}$ ou de la croyance.

9 Ces réserves faites, il me semble que, mon enquête portant sur une population jeune, en majorité estudiantine, de grandes villes (Athènes et Volos), la crédibilité des réponses doive être considérée avec un peu moins de méfiance, même si la prudence demeure de rigueur. La plus grande difficulté fut sans doute d'avoir eu, souvent, à interpréter telle ou telle réponse, d'essayer de comprendre ce qui pouvait bien se cacher derrière telle ou telle affirmation, notamment lorsque le questionné négligeait (ou ne voulait, ou ne savait ?) de répondre à la question « Pourquoi ?». Devint ainsi évident ce dont j'étais déjà plus ou moins convaincu : que les avantages du questionnaire anonyme étaient largement contrebalancés par ses inconvénients et que, surtout dans ce secteur culturel un peu particulier, seule une approche psychologique, voire psychanalytique, aurait eu 
quelque chance de se révéler éclairante. Ce qui, bien sûr, était exclu. Tout cela pour dire que ma modeste enquête ne saurait avoir d'ambition scientifique et qu'il ne s'agit, à mes yeux, que d'indications (avec toutes les marges d'erreur que l'ambigüité de certaines réponses introduit inévitablement) chargées d'illustrer mes réflexions ${ }^{16}$.

Si l'identité apparaît bien majoritaire, la profession de foi et l'orthodoxie des croyances, la fidélité au corpus sont loin de l'être, le Dieu des uns n'étant pas celui des autres. Les "croyants conformes" invoquent un Dieu personnel qui implique pratique et adhésion, alors que les déistes, même si l'appartenance à l'orthodoxie est revendiquée ${ }^{17}$, ne se réfèrent qu'à une sorte de Dieu impersonnel, pur "esprit", "force vitale", s'accommodant fort bien d'une incroyance face aux dogmes (miracles, vie éternelle), voire d'une indépendance totale vis-à-vis de l'Église. Ajoutons que, chez nos jeunes, cette affirmation de l'appartenance à l'orthodoxie peut fort bien s'accompagner d'une totale absence de foi, car il s'agit là d'une revendication d'appartenance nationale liée au besoin de conserver quelque chose d'essentiel de l'héritage culturel.

11 La participation religieuse elle-même n'a rien d'indicatif. Il faudrait sonder les coeurs et les consciences, mettre à nu les motivations, pour tirer quelque chose de significatif d'un simple chiffre comme celui, par exemple, de la fréquentation religieuse. Celle-ci ne dissimule-t-elle pas, très souvent, notamment dans les sociétés à fort concensus, c'està-dire à fortes pressions sociales, un athéisme caché, inavoué, voire ignoré de l'agent lui-même? G. Le Bras avait déjà remarqué que «la pratique individuelle est d'autant moins enracinée dans l'individu qu'elle est généralisée dans un groupe ${ }^{18}$. Inversement, la non-participation à la messe dominicale, ou autre cérémonie ou sacrement, et bien qu'on puisse la penser comme en rapport avec l'effritement général de la croyance religieuse, peut aussi s'accommoder d'une foi profonde, vécue individuellement et selon son propre cheminement. Ainsi, une pratique religieuse peut aussi bien traduire la foi que dissimuler l'absence de foi, alors que le refus d'une telle pratique permet de situer le sujet soit dans une catégorie religieuse opposée, soit dans une position non-religieuse. C'est le cas, dans notre pays, pour le baptême et le mariage, puisque ce sont ceux qui ne se marient pas à l'église ou qui ne baptisent pas leurs enfants qui, seuls, nous fournissent quelques informations sur l'évolution des croyances religieuses. Pratique et adhésion religieuses apparaissent ici quasiment indépendantes l'une de l'autre.

Enfin, je l'ai laissé transparaître plus haut, je ne suis pas loin de penser qu'en matière d'adhésion religieuse, toute typologie purement sociologique est, non seulement insatisfaisante, mais presque nécessairement sans valeur, car nous nous trouvons, dans ce domaine très particulier de la culture, face à un phénomène d'ordre fortement psychologique.

\section{Remarques sur les prises de position de l'Église orthodoxe grecque}

13 L'Église orthodoxe grecque apparaît plutôt discrète, au regard des deux autres branches du christianisme, sur les problèmes sociaux, et les diverses Encycliques du Saint Synode ne sont plus publiées systématiquement depuis 1956. Destinées aux pasteurs de l'Église qui doivent faire connaître la position de l'orthodoxie aux fidèles, ces textes ne sont, pour la plupart, communicables que sur demande écrite au Saint Synode. Les Encycliques ne sont publiées et destinées directement "au pieux peuple 
grec" que lorsque des questions considérées comme très importantes sont en jeu. Il en a été ainsi à propos de l'introduction du mariage civil, avec la publication de l'Encyclique 2309 de janvier 1982, reprise en substance par la bulletin officiel du Saint Synode, destinée à être distribuée dans les églises ${ }^{19}$. Il est intéressant de noter, à ce sujet, que la position de l'Église (acceptation du mariage civil, mais sans obligation) a été retenue par le texte du législateur. Il en a été de même lorsque les autorités ecclésiastiques entendirent mobiliser les fidèles contre un projet de loi de 1987 destinée à affaiblir la puissance temporelle de l'église (en intervenant dans la gestion de sa richesse immobilière, considérable) par la publication d'une lettre ouverte de 8 pages aux divers responsables politiques du gouvernement et de l'opposition ${ }^{20}$.

Cependant, la plupart du temps, les prises de position sont plus discrètes. C'est que l'intervention quotidienne de l'Église dans les affaires du monde est prise en charge, tout à fait efficacement à ce jour, par une foule de théologiens chargés de l'enseignement religieux (obligatoire dans les écoles et collées), par la création, depuis 1989, de stations de radio et chaînes de télévision contrôlées par l'Église, par un certain nombre de journaux pro-orthodoxes, souvent influents, ainsi que par les nombreuses "confréries", particulièrement actives et dont le caractère non-officiel permet de ne pas engager directement la responsabilité des autorités ecclésiastiques.

15 J'ai donc choisi une des revues des Confréries ( $\Sigma \omega \tau \eta ́ p$, l'une des plus importantes confréries orthodoxes avec $Z \omega \eta$ et $\Sigma \tau \alpha u \rho o ́ \varsigma)$ connue pour ses positions traditionnalistes et nationalistes, mais qui reprend souvent, avec plus de dynamisme sans doute, la

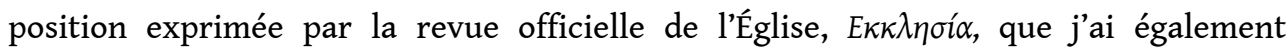
dépouillée, ainsi qu'un certain nombre de fascicules et publications officielles ou semiofficielles traitant de divers problèmes de nature sociale (et dont on retrouve l'écho dans les manuels scolaires d'éducation religieuse) pour illustrer le point de vue de l'Église orthodoxe grecque. Si ces prises de position semblent bien conservatrices et proches des vues des moines du Mont Athos (République théocratique de moines jouissant d'un statut autonome, et véritable centre spirituel de l'orthodoxie), c'est qu'en vérité l'influence de ceux-ci demeure considérable. Ajoutons que, si la revue $\Sigma \omega \tau$ rń n'a qu'un caractère semi-officielle, elle jouit du soutien évident du Saint Synode, et que le périodique destiné aux enfants diffusé par cette même revue depuis plus de 30 ans ${ }^{21}$ a obtenu du ministère de l'éducation et des cultes, depuis sa fondation jusqu'en 1982 (autorisation rétablie un court temps en 1989), le droit d'être diffusée dans les écoles. Bien que ce ne soit pas le rôle du sociologue de distribuer des brevets d'orthodoxie, force lui est de constater que cette revue est, elle aussi, tout à fait représentative des positions de l'Église officielle, tout comme, du reste, les divers fascicules publiés par la diaconie apostolique de l'Église de Grèce ${ }^{22}$. Par ailleurs, les tendances conservatrices sont incontestablement dominantes au sein de l'Église

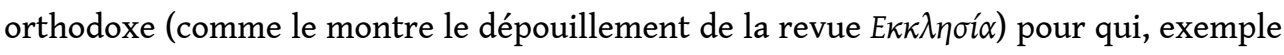
significatif, le protestantisme n'est déjà plus du christianisme.

\section{La situation juridique de l'Église orthodoxe de Grèce}

La mention, dans l'actuelle Constitution, de l'orthodoxie comme "religion dominante" doit être comprise, juridiquement, comme "religion de l'État", l'Église orthodoxe étant considérée comme l'Église de l'État, à caractère d'établissement public. Il en résulte que, si, d'un côté, l'État peut contrôler les actes des organes ecclésiastiques en ce qui 
concerne l'administration de l'Église, mais aussi les droits des citoyens, il est tenu, de l'autre, de la soutenir financièrement, et même en aidant à son prosélytisme au moyen de l'enseignement religieux, obligatoire dans les écoles et collèges.

Autre disposition en opposition avec les Droits de l'homme tels que les conçoivent les démocraties occidentales européennes: la mention de la religion sur les cartes d'identité. Cette mention, à laquelle l'Église demeure fermement attachée, que le gouvernement socialiste a lui même-même accepté de maintenir et dont seule une faible minorité des Grecs perçoit le danger ${ }^{23}$, est évidemment une disposition dont les partenaires européens devront, tôt ou tard, réclamer l'abolition. Les responsables religieux ont parfaitement perçu le danger et s'efforcent, tout en tentant de faire passer les partisans de l'abolition pour « des athées mécréants se mobilisant » contre l'Eglise ${ }^{24}$, de présenter cette mention comme un élément indissociable de l'identité nationale. Il n'est pas impossible, du reste, que l'actuelle mobilisation de l'Église contre la Convention de Schengen soit liée à cette question: risque, en effet, aux yeux des responsables orthodoxes, de plus grande circulation des hérésies et religions concurrentes, mais aussi celui que soit adopté le texte proposé par le gouvernement grec destiné à protéger la vie privée des citoyens et qui prévoit d'interdire la collecte des données à caractère confidentiel parmi lesquelles, bien sûr, la religion ${ }^{25}$.

Lorsque des décisions visant à une déchristianisation de la vie sociale et à un respect accru des consciences sont adoptées, il s'agit le plus souvent de compromis permettant à l'Église de maintenir ses positions. Ainsi du mariage laïc. Adopté en 1983 par le gouvernement socialiste, mais non imposé puisque, comme le réclamait l'Église, le mariage religieux conserve son caractère officiel, il ne pouvait, dès lors, être adopté que par un nombre réduit de jeunes, dans une société où les pressions sociales et familiales demeurent fortement dissuasives ${ }^{26}$, mais aussi où les diverses manifestations religieuses sont massivement suivies, tant par les incroyants que par les croyants car perçues comme des occasions, souvent uniques, de distraction et de fête. Et puis, et les réponses des jeunes interrogés sur ce point sont explicites, il est clair que le cérémonial civil est loin de comporter la même charge symbolique et émotionnelle que le rite religieux. Il est donc inutile de se demander pourquoi, par exemple, le baptême, nonobligatoire, demeure la règle quasi-absolue.

Cette place privilégiée de l'Église dans le pays a longtemps rendu l'État lui-même impuissant lorsqu'il s'agissait de prendre des mesures que celle-ci refusait, notamment dans le domaine du droit de la famille, l'autorité ecclésiastique jouissant d'une influence certaine en milieu rural. Or, c'est une grande partie de ces privilèges que l'intégration européenne et la perspective d'un véritable espace unique européen risquent de remettre en cause ${ }^{27}$. Cette inquiétude de l'Église orthodoxe s'exprime souvent sans ambigüité, même dans la presse quotidienne ${ }^{28}$. Une timide évolution vers un État laïc a quelque peu été amorcée par la Constitution de 1975 (actuellement en vigueur), même si dans ce domaine sensible des rapports entre l'Église et l'État, on ait préféré s'en tenir à des formulations archaïques, résultats des compromis adoptés. Ainsi, s'il n'est plus nécessaire que le chef de l'État soit orthodoxe, le texte de la prestation de serment, "au nom de la Trinité », reste celui des constitutions antérieures, et l'on peut penser que seule l'acceptation d'un conflit ouvert avec l'Église (avec tous les risques électoraux que cela impliquerait) pourrait, par exemple, amener les Partis à élire un non-orthodoxe au fauteuil présidentiel. 
20 Il convient cependant de noter qu'un débat est actuellement largement ouvert sur une réforme de la Constitution qui pourrait déboucher sur une nouvelle conception des rapports entre l'Église et l'État, ce qui inquiète au plus haut point le lobby orthodoxe qui, déjà préoccupée de ce que le personnel politique est de plus en plus éloigné des préoccupations de l'Église ${ }^{29}$, se bat bec et ongles pour «que l'on ne touche pas à la constitution pour tout ce qui a rapport avec les questions religieuses $»^{30}$.

\section{Christianisme, tolérance et démocratie : d'une religion allant de soi au choix personnel}

Incontestablement, en cessant d'être le principe organisateur et la référence ultime de la vie sociale et morale, en étant contrainte d'abandonner l'organisation du monde et de disputer à d'autres instances politiques, morales et juridiques ses normes et son éthique, le christianisme est menacé dans son essence même, puisque, de religion imposée, il devient religion choisie ou rejetée, puisque l'adhésion religieuse ne va plus de soi et devient décision personnelle. Et l'on peut douter que les combats menés par telle ou telle fraction de l'Église grecque pour maintenir intact le mythe de l'identification entre orthodoxie et grécité aient quelque chance d'aboutir à terme, tant la modernité est désormais partiellement coupée de ses racines religieuses.

Dans la plupart de nos sociétés occidentales, la majorité des jeunes chrétiens d'aujourd'hui se refuse à croire que leur religion serait la seule vraie, car il n'y a rien qui choque autant l'esprit moderne de relativisme qu'une institution qui affirme monopoliser le rapport à la vérité. Ce qui a conduit à "la fin des idéologies" est aussi ce qui entraîne le rejet, par ses fidèles mêmes, d'une Église omnisciente. Or les Églises trainent le pas et semblent avoir bien des difficultés à intégrer cette nouvelle attitude. Cette idée moderne d'un pluralisme dans l'Église est sans doute, comme le suggère un théologien catholique, à mettre en rapport avec la généralisation de la pensée démocratique ${ }^{31}$. Dès lors, les autorités de tutelle n'ont d'autres ressources que le double langage et, entre deux chaises, tentent de ne pas trop se discréditer : ni apprentis sorciers dilapidateurs de l'héritage, ni obscurantistes ${ }^{32}$.

En Grèce toutefois, où les réticences ont toujours été très fortes à se déclarer athée ${ }^{33}$, l'Église orthodoxe est beaucoup moins touchée par ce phénomène de relativisation, si répandu dans le monde occidental ${ }^{34}$. L'explication tient incontestablement à ce que l'orthodoxie est encore considérée comme une des racines de la grécité et, donc, comme un élément culturel qu'on ne saurait impunément dévaloriser ; sans doute aussi cela reflète-t-il l'efficacité de l'enseignement religieux obligatoire à l'école.

Cependant, pour les croyants même, il n'y a plus nécessairement de Vérité unique, de discours d'autorité acceptable, mais des possibilités multiples d'interprétations diverses des quelques textes demeurés authentiques mais interprétables, et cela hors de l'autorité. Ces conceptions et pratiques nouvelles qui croissent parallèlement à l'extension de la modernité, et qu'a analysé, par exemple, D. Hervieu-Léger ${ }^{35}$, je les retrouve en partie dans mon enquête (dont on trouvera un compte-rendu en annexe).

Dans les pays de tradition catholique, et même là où le poids de la tutelle chrétienne apparaissait le plus lourd, les interventions des autorités religieuses dans les affaires publiques au nom d'une certaine morale commencent à se solder par des échecs. Ce fut, par exemple, le cas de l'Italie à propos de l'avortement ${ }^{36}$ ou de l'Irlande pour le divorce. 
L'Église orthodoxe elle-même n'a pu s'opposer, comme elle avait pu le faire jusqu'à il y a environ une vingtaine d'années, à des mesures libérales concernant le droit des individus (contraception, avortement, mariage laïc, etc.), à l'introduction dans le pays de ce que l'Église grecque critique comme "modèle européen" venant mettre en cause "notre belle tradition de la famille ${ }^{37}$. Les réponses données par les jeunes de mon enquête reflète les réticences, même des orthodoxes conformes, à l'intervention de l'Église dans les questions de morale individuelle ${ }^{38}$, laquelle ne peut que déplorer «la dégradation morale de la jeunesse » et "son éloignement des principes et valeurs traditionnelles $»^{39}$.

En fait, les normes édictées par la tradition ont cessé désormais d'aller de soi, et l'on prend de plus en plus conscience que celles-ci peuvent s'opposer à l'éthique et que cette dernière repose sur d'autres bases que la règle imposée, qu'il s'agisse de l'éthique de la discussion («seule éthique qui convienne à une société démocratique ${ }^{40}$ ) ou de la responsabilité (telle que la concevaient des esprits aussi divers que Dostoievski, Weber ou Camus). En outre, en Grèce même, l'idée qu'orthodoxie et nation sont inextricablement mêlées ne va plus autant de soi, et l'on commence à percevoir que l'unité nationale peut reposer sur autre chose que des valeurs communes. Fortement minoritaire, c'est vrai, cette nouvelle tendance mérite cependant d'être relevée ${ }^{41}$.

\section{Orthodoxie et éducation}

Menace de taille aux yeux de l'Église grecque que les pressions, encore timides, mais que l'appartenance à l'Union européenne devrait tôt ou tard accentuer, contre l'obligation de l'enseignement religieux dans les écoles et collèges. C'est, bien sûr, qu'un enseignement sans religion, qui aurait évacué l'irrationnel au profit de la pensée et de la réflexion, apparaît menaçant pour le maintien de la tradition orthodoxe fondée sur d'autres éléments. En effet, comme le note R. Thauless, non seulement l'intelligence et le sens critique ne sont guère sollicités dans l'enseignement religieux, mais « l'usage abusif de techniques de suggestion comme méthode pastorale peut conduite à une surestimation de l'attitude d'adhésion passive $»^{42}$. C'est, du reste, ce que notent avec satisfaction les auteurs (enseignants et théologiens) d'une petite enquête sur les résultats de leur enseignement religieux dans leur école : «il semble clair que cette leçon influence directement l'adolescent plus qu'aucune autre ». Cette "efficacité" de la leçon de religion est résumée, de façon significative, par cette déclaration d'un des adolescents de l'enquête: "j'aime la leçon de religion parce que toutes mes incertitudes disparaissent $\aleph^{43}$.

Cela dit, il est bien entendu que cet enseignement religieux n'est pas seulement défendu par les théologiens, et les chrétiens en général, et que nombre d'incroyants mêmes sont tout prêts à en voir maintenir le principe, tant la religion et son enseignement leur semblent chargés d'efficace morale. Pourtant, l'article 16 de la Constitution grecque, qui déclare que l'éducation a pour but «le développement de la conscience nationale et religieuse " devrait, de toute évidence, être amputé de ce dernier terme pour être en accord avec l'idée de liberté de conscience et tous les textes internationaux s'y référant. Remarquons, au passage, que l'article 13 de la Constitution affirme que "le prosélytisme est interdit», sans qu'il soit nulle part précisé que le prosélytisme de l'Église orthodoxe ferait exception. 
29 En opposition donc avec les quelques textes européens en la matière, violant manifestement le droit des parents à donner à leurs enfants l'éducation de leur choix, cette obligation de l'éducation religieuse sera tôt ou tard objet de discussions à Bruxelles. En Grèce même, les esprits évoluent et (même si certains théologiens n'hésitent toujours pas à prôner une éducation purement orthodoxe pour nos jeunes, soutenant que le développement de la personnalité ne saurait se réaliser, dans notre pays, hors de l'appartenance à l'Église nationale ${ }^{44}$ ) les prises de position se multiplient en faveur d'une remise en cause de cette conception ${ }^{45}$. La Confédération des Instituteurs Grecs ( $\triangle$.O.E.) a ainsi proposé, en mai 1991, dans ses conseils au ministère, le remplacement du cours d'instruction religieuse par une histoire des religions ${ }^{46}$. Il en est de même de l'O $\Lambda \mathrm{ME}$ (Association syndicale des enseignants du secondaire) qui, elle aussi, réclame la suppression de l'obligation de l'enseignement religieux ${ }^{47}$. Du reste, une décision du Conseil d'État de 1995 (3356/ 1995) ouvre la voie dans cette direction puisqu'elle supprime l'obligation de suivre l'enseignement religieux pour les enfants d'une autre religion, les sans religion et les athées. Et il semble même (à la lecture, du moins, de ma petite enquête) que la majorité des jeunes s'y montreraient beaucoup plus favorables ${ }^{48}$ que leurs aînés qui, si on en croit une enquête de 1995, seraient massivement en faveur de l'obligation $(80,2 \%)^{49}$. Or, l'essentiel du prosélytisme de l'Église passe aujourd'hui, nous l'avons vu, par ce canal privilégié de l'école. D'où la

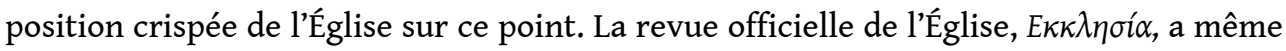
consacré une longue série d'articles (de février à décembre 1995) en critiques et réfutations d'un interview d'un professeur de droit constitutionnel et député européen où il se prononçait en faveur de la non-obligation ${ }^{50}$, et l'Union hellénique des Théologiens a écrit, en juin 1996, au ministre de l'Éducation nationale pour lui demander le maintien du cours de religion dans toutes les classes du secondaire. Il est vrai que, pour les diplômés de théologie (qui, souvent, n'ont pas vraiment choisi cette discipline), il n'est guère d'autre débouché professionnel que cet enseignement. En tout cas, aux yeux des orthodoxes militants, la seule position acceptable est celle-ci: «l'enseignement ne saurait être coupé de nos racines spirituelles " ${ }^{51}$.

\section{Orthodoxie et nation}

30 L'un des grands succès de l'orthodoxie, mais qui est aussi ce que le théologien orthodoxe français 0 . Clément considère, lui, comme «le péché historique majeur de l'orthodoxie moderne $»^{52}$, est, en Grèce, d'avoir su se présenter comme indétachable de l'idée nationale, comme sa principale racine, comme son indispensable pilier ${ }^{53}$. L'orthodoxie est ainsi considérée comme ayant été à la pointe de la guerre de libération nationale de 1821 (ce qui est faux, même si les prêtres qui, à titre personnel, prirent part aux soulèvements ne furent jamais excommuniés par leur Église ${ }^{54}$ ) et l'on insiste sans cesse sur son rôle de gardienne de la langue et de la culture nationales. Dès lors, aux yeux de la majorité des Grecs, Église et nation ne font qu'un, et l'intervention de la première dans la vie politique et sociale du pays ne choque guère, comme l'illustrent les réponses à mon questionnaire.

31 Ainsi, ce qui, ailleurs en Europe, va maintenant de soi (la désimbrication du politique et du religieux, la distinction entre nation et religion) relève, en Grèce, presque de la subversion, la conscience nationale étant perçue, pour une bonne part, sous le signe du 
religieux $\mathrm{x}^{55}$. Ce relatif consensus sur l'orthodoxie s'explique surtout par une quête d'identité et le refus d'une altérité menaçante ou supposée menacée.

En Occident, le Pape invoque le christianisme au nom des racines culturelles, ce " patrimoine chrétien si profondément inscrit dans la culture européenne " ${ }^{56}$. C'est déjà ce que disait Paul VI : «nous pensons, nous, que seule la civilisation chrétienne, dont est née l'Europe, peut sauver ce continent du vide qu'il éprouve ${ }^{57}$. En Grèce, l'Église lie la grécité à l'orthodoxie. Et, de même que, dans les discours du Pape, l'unité de l'Europe serait menacée par la déchristianisation, pour l'Église grecque, l'unité nationale serait en danger dès lors que la présence de l'orthodoxie serait limitée.

Apparemment de même nature, ces deux tendances se séparent sur plus d'un point. S'il n'y a pas vraiment (pas encore ?) de conscience européenne, et donc pas de patriotisme européen, le sentiment d'identification à la nation est, bien sûr, profondément ancré chez les Grecs, ce qui permet à l'orthodoxie de pouvoir apparaître (dans la perception, quelque peu obsessionnelle ici, d'un climat de menaces) comme un ciment essentiel de l'unité nationale. En ce sens, on pourrait dire que la force de l'Église grecque lui vient justement de ce qui apparaît un peu comme sa faiblesse: son caractère local, que l'échec de l'œcuménisme renforce encore, même si l'évolution en Europe de l'est et dans les Balkans pourrait lui ouvrir des perspectives nouvelles.

$\mathrm{Si}$, depuis longtemps, l'orthodoxie a dû abandonner les rênes du pouvoir aux politiciens, son influence, on l'a vu, demeure considérable sur la vie civile et les politiques menées au nom de la nation. C'est un secret de Polichinelle que nombre de députés appartiennent, plus ou moins explicitement, au lobby orthodoxe, et il n'est que de feuilleter les textes des débats à l'Assemblée nationale pour s'en convaincre. On peut même être étonné de la façon dont les ministres les plus "laics" (ce terme, dans le sens français, n'est pas traduisible en grec et n'a pas vraiment d'équivalent ${ }^{58}$ ), pourtant visiblement majoritaires, obtempèrent souvent aux injonctions de ce lobby. C'est, sans doute, que l'activisme "sécularisateur" est mal venu et vaguement assimilé à l'extrêmisme gauchiste.

35 C'est aussi à un autre niveau que se manifeste la présence de l'Église, là où se situe une part essentielle de son magistère: le niveau symbolique. Inutile de rappeler ici toute l'importance que revêt cet aspect dans la réalité du pouvoir. Ainsi, si l'Église n'exerce plus aucun pouvoir politique, c'est elle qui parait encore le déléguer. Qu'il ne s'agisse que de symbole, qu'importe; l'essentiel est que, par la cérémonie d'investiture des membres du gouvernement et des députés, élus par les citoyens mais prêtant serment sur l'Évangile, recevant la bénédiction ecclésiastique et jurant sur la Sainte Trinité (article 59 de la Constitution), l'orthodoxie affirme sa présence comme instance de légitimation.

36 Sans doute l'Église comprend-elle peu à peu que la sécularisation de la société grecque est inévitable à terme et entend-elle se maintenir exclusivement, mais fermement : ce qui explique son attachement inébranlable à l'obligation de l'enseignement religieux dans les écoles, dans le domaine du contrôle des consciences, tout en entendant continuer d'intervenir directement dans la politique. Elle ne peut, en effet, se payer le luxe d'une distanciation trop grande d'avec la sphère étatique, ne pouvant avoir l'ambition (contrairement à sa grande sœur-rivale catholique ou à l'Islam) de rassembler, sinon l'humanité entière, du moins une grande partie de celle-ci. Certes, la nouvelle situation dans les pays de l'Europe de l'est et dans les Balkans pourrait lui offrir un champ d'expansion inespérée. Ainsi le soutien sans faille aux nationalistes 
serbes lors de la "guerre civile" en ex-Yougoslavie peut-il être lu, aussi, sous cet angle. Mais il est loin d'être sûr que la situation soit aussi favorable pour le nationalisme panorthodoxe grec qu'on le croit souvent. Et ce ne sont pas les tensions périodiques avec le patriarche russe, par exemple, qui s'inscriront en faux contre cette hypothèse. Quoi qu'il en soit, trop liée en fait à l'espace national (la "grécité"), l'Église orthodoxe grecque apparaît profondément dépendante du temporel.

Cependant, il est bien évident que la séparation du temporel et du spirituel, dans les limites où cela est possible, ne saurait conduire, dans cet Occident jadis ennemi mais dont, désormais, la Grèce est partie intégrante et partage le destin, qu'à une suprématie du premier. En effet, contrairement à ce qui se passe dans d'autres régions du monde, en Inde, par exemple, où la religiosité est tellement intégrée à la quotidienneté que la séparation des deux sphères n'a nullement conduit à la perte de suprématie du spirituel ${ }^{59}$, nos sociétés sont devenues trop "temporalisées" pour que la séparation de l'Église et de l'État ne soit pas perçue par les autorités ecclésiastiques, et avec raison, comme une menace sur leur magistère politico-culturo-moral.

Quelques chiffres sur une enquête sur 607 jeunes (grande majorité : étudiants de l'Université d'Athènes et de Solos) entre novembre 1991 et février 1992.

1) Orthodoxes "conformes"; $26,35 \%$ (le pourcentage de l'enquête publié par la revue Epsilon - décembre 1991 - est de 26,7\%).

Athées convaincus : $6 \%$ (le pourcentage de l'enquéte publiée par Epsilon est de $6,6 \%$ ).

Les autres allant de l'orthodoxie non-conforme à l'athéisme convaincu, la plupart (28,8 \%) pouvant être considérés comme "déistes" (voir plus bas, l'"histogramme de l'appartenance religieuse"). Ajoutons que pour $42,83 \%$ des jeunes interrogés, l'orthodoxie est "le vrai christianisme" et que la croyance aux horoscopes $(15,32 \%)$ peut fort bien accompagner l'orthodoxie la plus conforme.

2) En ce qui concerne les principaux phénomènes perçus comme menaçant l'identité nationale (19,7\% pensent qu'il n'y a pas menace), l'américanisation vient en tête avec 30,6\%, suivie de l'intégration européenne (20,75\%), de la libéralisation des moeurs $(16,47 \%)$ et de l'athéisme (10,7\%). Ajoutons à ces chiffres les 7,9\% de réponses mentionnant la «rupture des racines et de la tradition» et la "xénomanie".

3) Parmi les jeunes interrogés par questionnaire sur la question de savoir si la religion doit continuer d'être enseignée à l'école, $38,55 \%$ répondent «oui, obligatoire », 40,36 \% souhaiteraient que cet enseignement soit facultatif tandis que seuls $1,21 \%$ répondent que cet enseignement devrait être supprimé.

D'autre pari, $56,5 \%$ seraient favorables au remplacement de la leçon de religion par un cours d'histoire des religions tandis que $29,43 \%$ s'y déclarent opposés.

4) Pour ce qui est du rapport perçu entre science et religion, $39 \%$ considèrent que la science complète la religion, alors que $13,83 \%$ pensent qu'elle se trouve en opposition avec elle et $35,58 \%$ qu'elle la démythifie.

5) À la question de savoir si l'Église doit intervenir dans les grands débats de société (la plupart le pense) et dans lesquels, une très forte minorité attend de l'église qu'elle se mobilise surtout contre la pauvreté et les inégalités sociales (67,7\%). Les autres causes où l'Église devrait intervenir sont beaucoup moins évoquées: la contraception (15,48 \%), la conception in vitro $(15,15 \%)$ et l'avortement $(24,7 \%)$.

6) Assez peu de jeunes interrogés considèrent comme inacceptable le fait que l'orthodoxie jouisse, d'après la Constitution, d'un statut privilégié par rapport aux autres religions $(19,27 \%)$, tandis que $22,89 \%$ trouvent cela juste. Notons que la réponse «logique » choisie par la majorité d'entre eux $(49,9 \%)$ peut aussi bien signifier « logique mais regrettable » que « logique et acceptable ».

Que les membres du gouvernement et les députés prêtent serment « au nom de la Sainte Trinité » est considéré comme « juste » par 26,19\% des interrogés tandis que 
$27 \%$ y sont opposés, considèrant cela comme "inacceptable » $(9,55 \%)$ et « ridicule» $(18,45 \%)$. Ici encore, la réponse «logique» peut difficilement s'interprêter.

Enfin, en ce qui concerne la mention de la religion sur la carte d'identité, on sera peut-être surpris de noter que seuls $9,88 \%$ des jeunes interrogés la jugent « dangereuse » et $17,79 \%$ « inacceptable », tandis que $27 \%$ considèrent cela comme « juste » et $37,39 \%$ comme « logique ».

Sentiment de l'appartenance religieuse et croyance

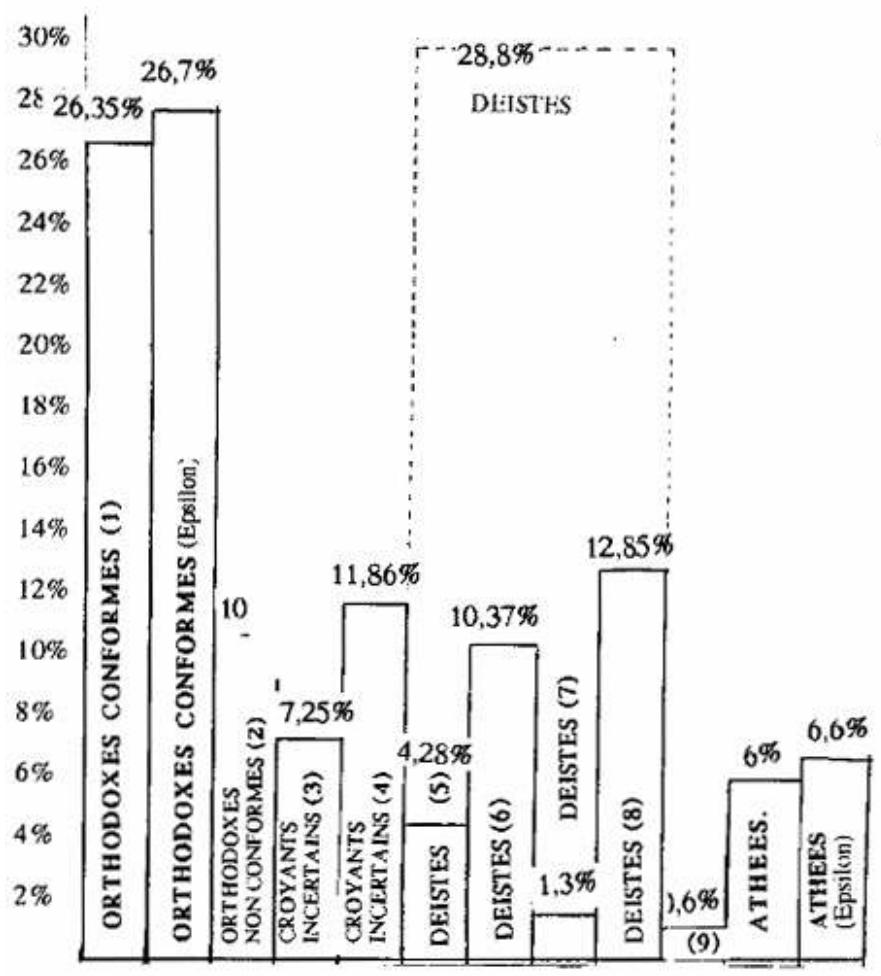

(1) Croient en Dieu, aux miracles et à la vie éternelle. Se sentent membres d'une Église. Pratiquent la prière.

(2) Croient en Dieu, aux miracles et à la vie éternelle (soit ne prient jamais, soit ne se sentent pas membres d'une Église, soit les deux).

(3) Croient en Dieu et aux miracles, mais pas à la vie éternelle ; ou croient en Dieu et à la vie éternelle, mais pas aux miracles. Se sentent membres d'une Église et pratiquent la prière

(4) Croient en Dieu et aux miracles (mais pas à la vie éternelle) ou en Dieu et à la vie éternelle (mais pas aux miracles). Soit ne se sentent pas membres d'une Église, soit ne prient jamais, soit les deux.

(5) Ne croient qu'en Dieu (ni aux miracles, ni à la vie éternelle). Se sentent membres d'une Église et pratiquent la prière.

(6) Ne croient qu'en Dieu (ni aux miracles, ni à la vie éternelle). Ne se sentent pas membres d'une Église ou ne prient jamais (ou les deux).

(7) Croient en une Force supérieure. Se sentent membres d'une Eglise. Pratiquent la prière.

(8) Croient en une Force supérieure. Ne se sentent pas membres d'une Église ou ne prient jamais (ou les deux)

(9) Ne croient qu'aux horoscopes. 


\section{NOTES}

1. On trouvera en annexe un rapide compte-rendu des réponses qui met en évidence combien les croyances religieuses de ces jeunes, quand bien même ils se diraient orthodoxes, sont relativement éloignées du corpus de croyances de l'Église.

2. «La grande majorité de la population grecque (...) considère l'orthodoxie comme une partie de l'identité culturelle collective (...). Elle est attachée à l'orthodoxie comme ensemble culturel général et non comme croyance religieuse particulière » (Makrides (Vassilios), «Orthodoxy as a Condition sine qua non : Religion and State. Politics in Modern Greece from a Socio-Historical Perspective ", Ostkirchliche Studien, 40, Heff 1991, pp. 301-302).

3. Ibid., p. 304.

4. En poussant les choses à l'extrême, on pourrait dire que cette identification Église/État, qui demeurera une constante pendant quasiment toute la période byzantine, remonte à l'installation de Justinien à Constantinople.

5. Savatou (K. Chrisostomou, archimandrite), «La présence de l'Église orthodoxe dans l'Europe

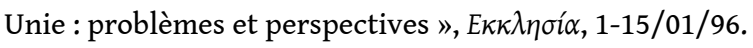

6. Ahrweiller (Hélène), L'idéologie politique de l'Empire byzantin, Paris, 1975, p.79, 107.

7. Ducellier (Alain), Chrétiens d'Orient et Islam au Moyen-Age (VIIe-XV eme siècles), Paris, 1996, p.15.

8. Il est à souligner que la Constitution de 1975, actuellement en vigueur, a fait un pas en avant par rapport aux constitutions antérieures puisque ce n'est plus «le prosélytisme contre la religion dominante » qui est interdit, mais, en principe, toute forme de prosélystisme.

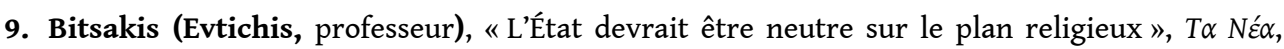
19/05/95.

10. Nombreux sont encore ceux qui ignorent que l'enfant peut être déclaré sous un nom sans passer par la cérémonie du baptême.

11. Notamment, Encyclique $2550 \mathrm{du}$ Saint Synode (12 mai 1993). Reprise de l'Encyclique $2548 \mathrm{du}$

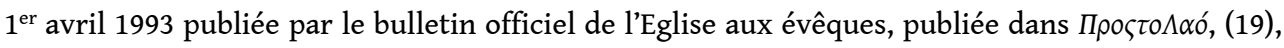
mai 1993, et destinée à être lue dans les églises.

12. Cette mention de la religion est notamment interdite par l'article 6 de la Convention du Conseil de l'Europe pour la protection des individus de janvier 1981.

13. Nissiotis (N.), "L'Église et la société dans la théologie orthodoxe grecque », Église et Société, Genève, 1966, t. 1, p. 68, cité par Tzanimis (Anastase), «La sociologie de la religion en Grèce ", Social Compass, 22 (1), 1975.

14. Alors même que l'occupation ottomane ne s'est guère souciée, semble-t-il, de combattre les cultures des peuples soumis. Sauf, justement, lorsque le "lobby grec" l'y poussait: c'est l'exception notoire, dont m'informe aimablement le professeur Alain Ducellier, du cas de l'Albanie qui ne put jamais utiliser sa langue par écrit, et où l'on ferma, en 1886, l'école albanaise de Korça à la demande du métropolite (grec). Je remercie ici Monsieur Ducellier pour l'ensemble de ses remarques et informations auxquelles cet article doit beaucoup.

15. Ainsi, la classification utilisée naguère par G. Le Bras (Bras (G. Le), Introduction à l'histoire de la pratique religieuse, Paris 1942), fondée sur les catégories "conformistes saisonniers" et "observants réguliers", n'aurait guère de sens dans le village grec où le lieu de rassemblement traditionnel est la place devant l'église et où les occasions privilégiées, sinon uniques, de rencontre et de fête restent les cérémonies religieuses.

16. J'avoue cependant avoir été quelque peu rassuré sur la valeur de ma petite enquête à la lecture d'un sondage (dont j'ignorais, jusqu'il y a peu, l'existence) effectué à peu près à la même date, portant sur un échantillon représentatif de la population athénienne et publié, en décembre 1991, dans le supplément hebdomadaire du journal Elevtherotypia. En matière de 
croyance, et là où les questions sont identiques aux miennes, les pourcentages sont quasisimilaires. Ce qui ne laisse pas, du reste, de surprendre; je me serais attendu, en effet, à un certain décallage en raison des différences d'âge et de niveau éducatif des deux groupes de sondés. Preuve que les conceptions mentales évoluent encore plus lentement qu'on ne le pense habituellement ou alors, s'agirait-il d'une revendication, chez mes jeunes étudiants, des racines supposées menacées?

17. $45,6 \%$ des jeunes de mon enquête.

18. Cité par Desroches (Henri), Sociologies religieuses, Paris, 1965, p. 42 note 1.

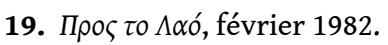

20. Lettre du Saint Synode de juillet 1987. Voir également le texte sur le même sujet destiné aux

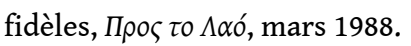

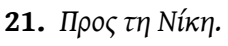

22. Il s'agit de l'organisme chargé des publications de l'Église et de la formation du Clergé.

23. $9,88 \%$ seulement des jeunes de mon enquête considèrent cette mention comme dangereuse.

24. $\Sigma \omega \tau \eta ́ \rho, 30 / 07 / 97$.

25. Le juriste grec Aristide Manesis, par exemple, écrit : «La protection constitutionnelle de la liberté religieuse entraîne le droit de déclarer, mais aussi, à l'opposé, de ne pas dévoiler ses croyances religieuses ou non religieuses. Ainsi l'obligation de déclarer ses convictions religieuses s'oppose-t-elle à la liberté constitutionnelle de la conscience religieuse » (Manesis (Aristide),

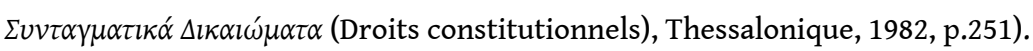

26. $14 \%$ de mariages civils à Athènes en $1983,9 \%$ en 1990 . Comme on le voit, ce pourcentage se réduit, et il est, bien sûr, beaucoup plus bas dans le reste du pays. Voir l'article de Ioanna Madrou dans le journal To Bń $\alpha$ du 01/12/91.

27. On pourrait, certes, évoquer la situation de l'Irlande qui montre qu'après 20 ans d'appartenance aux Communautés Européennes, la remise en cause de la cléricalisation de la société ne va pas de soi. Toutes les écoles y sont religieuses, l'avortement y demeure interdit par la Constitution, et le divorce vient seulement d'être accepté; de même, la vente libre des préservatifs ne fut adoptée qu'en 1985 et par deux voix seulement de majorité. La comparaison serait donc tentante, si ce n'était que la situation grecque est profondément différente, en ce sens que les lois grecques concernant la famille sont parmi les plus progressistes d'Europe. Sans doute parce que l'Église grecque intervient traditionnellement beaucoup moins, comme on l'a dit, que sa sœur catholique dans la vie privée des gens.

28. Ainsi l'influent journal conservateur, $K \alpha \theta \eta \mu \varepsilon \rho v v \eta ́$, dénonce-t-il, de temps à autre, «la propagande contre notre pays en vue d'une déchristianisation de la Constitution » (12/11/93).

29. Chez beaucoup de nos hommes politiques, « le respect envers l'Église orthodoxe s'est affaibli. Ce phénomène pose de nouveaux problèmes aux relations actuelles entre l'Église et l'État »:

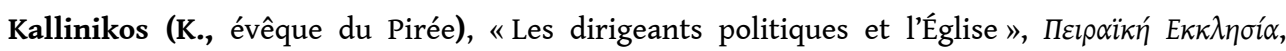
décembre 1990.

30. Kalokaitinou (Grégoire), "Que le peuple décide pour les relations avec son Église",

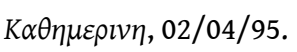

31. Marlé (René), « La théologie admet-elle le pluralisme ? », Etudes, décembre 1989.

32. C'est, par exemple, ce qui explique qu'une récente déclaration du métropolite de Zakinthos (candidat potentiel à la succession de l'Archevêque de Grèce) en faveur des relations sexuelles prémaritales (à une revue quelque peu "osée", c'est vrai : Penthouse, janvier 1998) ait pu faire couler tant d'encre et de salive: laissant entendre que la morale chrétienne ne serait ni universelle ni intemporelle, la petite phrase en a choqué plus d'un. Or, il faut sans doute l'inscrire dans cette stratégie d'adaptation qu'il est difficile à l'Église orthodoxe de ne pas adopter si elle entend se maintenir chez les jeunes. 
33. Ainsi, au recensement de 1920 (où, pour la première fois, on pouvait se déclarer "sans religion") on ne compta que 38 athées; 117 en 1928 et 121 en 1951. C'est dire que le chiffre d'aujourd'hui (696) peut paraître élevé.

34. $42,83 \%$ des jeunes de mon enquête (fussent-ils non traditionnalistes, voire déistes) considèrent l'orthodoxie comme le vrai christianisme.

35. Hervieu-Léger (Danièle), «Les manifestations contemporaines du christianisme et la modernité ", Christianisme et modernité, Paris : Centre Thomas More, Paris 1990 (Actes du colloque Christianisme et modernité organisé au centre Thomas More en septembre 1987).

36. $67 \%$ des Italiens approuvant par référendum la loi sur la libéralisation de l'avortement malgré l'intervention directe du Pape.

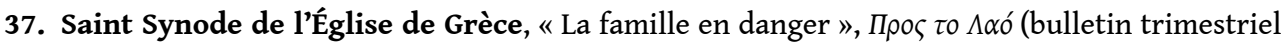

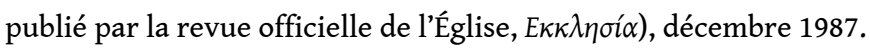

38. Répétons que l'Église orthodoxe intervient traditionnellement beaucoup moins dans la vie privée des fidèles que le catholicisme, sans doute parce que l'idée d'une relation immédiate et directe de l'homme avec Dieu rend le rôle de médiateur et de guide de l'Église beaucoup moins essentiel. Voir, par exemple, Ducellier (Alain), Le drame de Byzance. Idéal et échec d'une société chrétienne, Paris, 1976, p. 209.

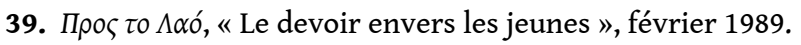

40. Ladrière (Paul), « L'éthique, soi et les autres », Informations Sociales, (9), 1991.

41. Que l'on me permette ici une parenthèse. Dans le souci, somme toute respectable, de ne pas apparaitre comme aveuglés par le parti pris, les fondateurs de la sociologie moderne se sont parfois embourbés dans une anthropologie fonctionnaliste de l'irrationnel bien discutable. « C'est en effet, écrit notamment E. Durkheim, un postulat essentiel de la sociologie qu'une institution humaine ne saurait reposer sur l'erreur et sur le mensonge : sans quoi elle ne saurait durer » (Durkheim (Émile), Les formes élémentaires de la vie religieuse, Paris, 1960, p. 3). Durkheim fait ici allusion à l'institution religieuse que le temps aurait soumis, à ses yeux, à une sorte de sélection naturelle de type darwinien. Or, le relativisme moral selon lequel les règles et les normes que "se donne" une société seraient bonnes pour elle, qu'elles seraient nécessairement correctes et qu'aucun jugement relatif ne serait possible sur la suprématie éthique de telle ou telle croyance, règle ou comportement est, à mes yeux, une position inacceptable.

42. Thauless (Robert H.), An Introduction to the Psychology of Religion, Cambridge University Press, 1971, p. 65.

43. Perraki (Jean), Konstantelou (Elevsi), «La religiosité de l'adolescent (enquête dans une

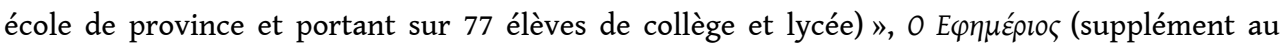
périodique $E \kappa \kappa \lambda \eta \sigma \iota \alpha), 01-15 / 08 / 93$.

44. Voir, par exemple, Repakis (Erakles), « La réforme de l'éducation religieuse en Grèce », To

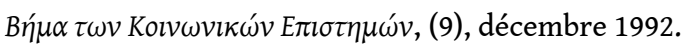

45. Par exemple, Bitsakis (Evtichis), art.cit. De même, le juriste grec, Aristide Manesis, dénonce cette relativité de la protection constitutionelle de la liberté religieuse, conséquence de la non-séparation de l'Église et de l'État qui s'exprime dans l'enseignement obligatoire du cours d'enseignement religieux dans les écoles (op.cit., pp. 257-258).

46. Ce à quoi est opposée l'Union nationale des Théologiens qui considère que cette leçon «ne doit pas devenir Religiologie »: lettre au ministre de l'Éducation en novembre 1997, citée par $\Sigma \omega \tau \eta ́ \rho, 19 / 11 / 97$.

47. Voir l'article du secrétaire de cette organisation dans $E \lambda \varepsilon v \theta \varepsilon \rho \circ \tau v \pi i \alpha, 01 / 10 / 95$ : « Religion et école ".

48. $56,5 \%$ favorable à une telle mesure. Et $40,36 \%$ favorable à un enseignement facultatif.

49. $T \alpha \alpha \varepsilon ́ \alpha, 19 / 05 / 95$.

50. Professeur Dimitri Tsatsos à $E \lambda \varepsilon v \theta \varepsilon \rho 0 \tau v \pi i ́ \alpha, 22 / 01 / 95$. 


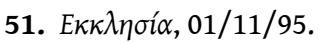

52. Clément (Olivier), "L'Église orthodoxe ", La Religion, dictionnaire du savoir moderne, Lille, 1972.

53. On peut sans doute rapprocher le cas grec du cas polonais où l'identification du sentiment religieux au sentiment patriotique est, là aussi, fondamental, l'Église étant considérée avoir été « la garante de la polonité » en « maintenant l'idée de souveraineté nationale et son expression première, la langue » : « Polonais=catholique est aussi une équation qui a marqué profondément la conscience nationale polonaise» (Offredo (Jean), «Le catholicisme polonais entre le traditionnalisme et les interpellations du monde moderne », Le Monde Diplomatique, mars 1978).

54. En effet, jouissant, sous la turcocratie, de considérables privilèges, ayant en charge l'ensemble du millet (ethnie) des orthodoxes, quelle que soit la nationalité de ceux-ci, l'Église pouvait, à juste titre, craindre, dans un premier temps, de voir les libérations nationales remettre en cause ses immenses privilèges, son incomparable influence et son œcuménisme. D'autant plus que les idées des Lumières qu'elle n'avait cessé de pourfendre et qui inspiraient, en partie, les révolutionnaires grecs, n'étaient pas pour la rassurer. Pour une étude sérieuse et documentée des idées des Lumières en Grèce à la fin du XVIII ème siècle, voir Kitromilidis (Michel), Les lumières néohélléniques, Athènes, 1996.

55. On n'oubliera pas que, lors de l'échange imposé de population entre la Grèce et la Turquie, en 1923 , le critère ne fut pas la langue mais la religion.

56. Jean-Paul II, «Europe, retrouve-toi toi-même », L'appel de Saint Jean de Compostelle, Paris : La Documentation Française, (1841), 05/12/82.

57. En 1975, aux évêques d'Europe. Cité par Poulat (Émile), «Jean-Paul II et l'Europe Chrétienne ", Le Genre Humain, (23), printemps 1991, p. 65.

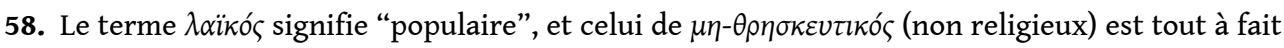
neutre. Mais sans doute cette remarque sur la laïcité est-elle fortement teintée d'ethnocentrisme puisque, au fond, on peut considérer cette notion française comme plutôt exceptionnelle. En ce sens, ce n'est pas le cas grec qui constitue une anomalie historique, mais la laïcité française.

59. Voir Dumont (Louis), Homo hierarchicus : le système des castes et ses implications, Paris, 1979. On pourrait, certes, évoquer le cas de la Turquie, société où le pouvoir politique est laïc mais où la population est, dans son écrasante majorité, profondément musulmane. Cependant, il faut souligner que la situation est très sensiblement différente puisque "l'édification de l'identité nationale turque s'est faite hors de l'Islam, voire contre lui » (Coulongou (Hélène), "Turquie : l'Islam, le nationalisme et la laïcité ", L'Histoire, avril 1998). Et, si on observe effectivement depuis quelques années une très forte montée de l'islam politique, on évaluait encore, il y a dix ans, que seule une très faible minorité de Turcs (7\% selon Gokalp (Altan), L'Histoire, avril 1998) réclamait le retour à la loi islamique.

\section{RÉSUMÉS}

L'Église orthodoxe grecque se sent menacée par le phénomène de déchristianisation. Bien que toujours croyante, la jeunesse grecque est de moins en moins pratiquante. De plus, la Grèce appartient à l'UE, où la plupart des États sont laïcs. Que l'orthodoxie soit la religion d'État, qu'elle soit indissociable de l'identité nationale grecque mettent en exergue aussi bien ses forces que ses faiblesses, notamment face à la sécularisation de la société. 
AUTEUR

JACKY PRUNEDDU

Historien et sociologue. 